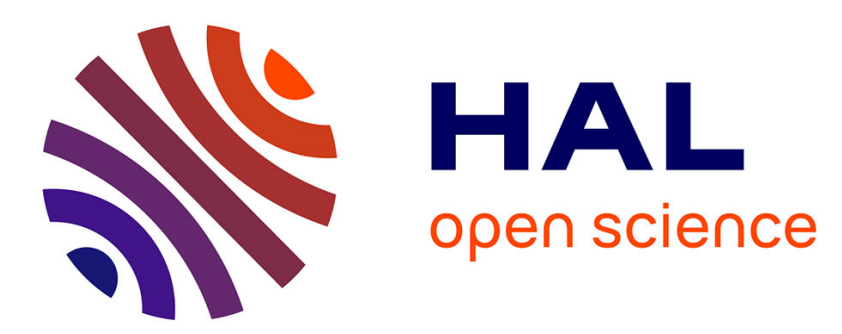

\title{
An optimization model and genetic-based matheuristic for parking slot rent optimization to carsharing companies
}

Stefano Carrese, Fabio d'Andreagiovanni, Tommaso Giacchetti, Antonella Nardin, Leonardo Zamberlan

\section{To cite this version:}

Stefano Carrese, Fabio d'Andreagiovanni, Tommaso Giacchetti, Antonella Nardin, Leonardo Zamberlan. An optimization model and genetic-based matheuristic for parking slot rent optimization to carsharing companies. Research in Transportation Economics, 2020, 85, pp.100962. 10.1016/j.retrec.2020.100962 . hal-02946279

\author{
HAL Id: hal-02946279 \\ https://hal.science/hal-02946279
}

Submitted on 11 Dec 2020

HAL is a multi-disciplinary open access archive for the deposit and dissemination of scientific research documents, whether they are published or not. The documents may come from teaching and research institutions in France or abroad, or from public or private research centers.
L'archive ouverte pluridisciplinaire HAL, est destinée au dépôt et à la diffusion de documents scientifiques de niveau recherche, publiés ou non, émanant des établissements d'enseignement et de recherche français ou étrangers, des laboratoires publics ou privés. 


\title{
An optimization model and genetic-based matheuristic for parking slot rent optimization to carsharing ${ }^{\star}$
}

\author{
Stefano Carrese $^{\mathrm{a}}$, Fabio D’Andreagiovanni ${ }^{\mathrm{b}, \mathrm{c}, *}$, Tommaso Giacchetti ${ }^{\mathrm{a}}$, \\ Antonella Nardin ${ }^{\mathrm{a}}$, Leonardo Zamberlan ${ }^{\mathrm{a}}$ \\ ${ }^{a}$ Dipartimento di Ingegneria, Universitá degli Studi Roma Tre, Via Vito Volterra, 62, \\ 00146 Roma, Italy \\ ${ }^{b}$ National Center for Scientific Research (CNRS), France \\ ${ }^{c}$ Sorbonne Universités, Université de Technologie de Compiègne, \\ CNRS, Heudiasyc UMR 7253, CS 60319, 60203 Compiègne, France
}

\begin{abstract}
Carsharing represents a major example of smart mobility service that allows a customer to rent a vehicle for a limited amount of time paying a per-minute fee. It may relieve people of the costly and non-sustainable burden of owning a car, especially when residing in a city. Though the spread of carsharing may bring significative benefits to (smart) cities, its penetration can be obstructed by nonup-to-date regulations, which can be still tied to a non-smart vision of mobility. In this study, we provide an overview of remarkable city regulations for carsharing, particularly highlighting the importance that parking policies can have in favouring the diffusion and use of carsharing services. Given such importance, we characterize the optimization problem of a local government that wants to analytically choose the best subset of parking slots to rent to carsharing companies, in order to improve urban mobility. To model and solve the problem we propose a new Binary Linear Programming problem and genetic-based matheuristic. Finally, we present results from computational tests referring to realistic data of the Italian city of Rome,
\end{abstract}

* This paper is an extended, improved version of the paper "An optimization model for renting public parking slots to carsharing services" published in Transportation Research Procedia vol. 45, pp. 499-506 (2020), as part of the Proceedings of the AIIT 2nd International Congress on Transport Infrastructure and Systems in a changing world (TIS ROMA 2019 - see Carrese et al. 2020).

*Corresponding author

Email addresses: stefano.carrese@uniroma3.it (Stefano Carrese), d.andreagiovanni@hds.utc.fr (Fabio D'Andreagiovanni), tommaso.giacchetti@uniroma3.it (Tommaso Giacchetti), antonella.nardin@uniroma3. it (Antonella Nardin), leonardo.zamberlan@uniroma3.it (Leonardo Zamberlan) 
showing that our optimization approach can return a fair territorial distribution of the parking slots, satisfying various families of constraints limiting the distribution.

Keywords: Shared Mobility, Carsharing, Parking Slot Renting, Optimization, Matheuristic.

\section{Introduction}

In recent times, smart mobility systems have attracted a lot of attention, since they are considered a fundamental component of modern smart cities, as recognized by national and international establishments and by major companies active in the landscape of digital economy (e.g., Benevolo et al. 2016; EU-INEA 2017). A Smart Mobility (SMOB) system can be defined as a strongly Information and Communications Technology (ICT)-supported Transport System (e.g., GeSi-ACN 2015; Kenny 2013). ICT is a crucial component of SMOB systems, which enables a continuous connection between the system administrators, the customers/users and the mobile and fixed infrastructures. Furthermore, it represents a key building block for offering innovative trustable and sustainable ways to move in urban and extra-urban scenarios. A major example of SMOB is represented by carsharing services. Nowadays, carsharing is intended as a mobility service that allows a user to rent a car for very short period of times (e.g., a few minutes) using a smartphone application and paying a per-minute fee (Weikl and Bogenberger, 2013). Such services have contributed to revolutionize urban mobility in the last decade, basing their success also on the strong diffusion of smartphones.

It is now widely recognized that carsharing and other SMOB systems can have a (very) positive impact on the quality of life in urban and extraurban scenarios, sensibly reducing the negative sides of transport systems (e.g., pollution caused by traffic, road congestion). For an overview of the benefits of SMOB, we refer the reader to (Benevolo et al., 2016; Bencardino and Greco, 2014; Carrese et al., 1996, 2017, 2020; Hessel, 2015; Lyons, 2016). Some studies have tried to precisely assess this positive impact. For example, Martin and Shaheen (2011) showed that the introduction of carsharing in North America led to a decrease in the average number of vehicles per family from 0.47 to 0.24 and that, on average, each shared car substituted from 9 to 12 private cars. Besides the positive impact on environment and economy, carsharing and other SMOB systems have a positive social impact too. Indeed, a considerable part of the costs that road transportation entails does not appear as internal costs borne by the drivers, but is taken into 
account as external costs (e.g., environmental impact) borne by the collectivity. Such external costs represent a third of the total and about $90 \%$ of them is due to private car owners (Lombard et al., 2005). Within this context, carsharing and other SMOB systems present features that can ease the internalization of costs, since they support the passage from privately-owned cars to mobility as a service, based on the concept of pay-as-you-go.

Though their benefits are pretty evident and clear, SMOB systems have found difficulties in being implemented. This is due to various reasons, such as: 1) the presence of regulatory frameworks that are often confused and not up-to-date for welcoming new innovative SMOB digital platforms and services; 2) the inertness of policy makers, which may ineffectively support the expansion of SMOB services, thus dooming them to remain just at an experimental and very low-scale level.

In recent years, among SMOB systems, carsharing has attracted particular attention and has been the subject of intense research from many point of views. Recent research studies have been aimed at better quantifying the benefits of carsharing, such as how it contributes to reduce the need for a private car (Becker et al., 2018) and how it favours the penetration and adoption of electric vehicles (see e.g., Meisel and Merfeld 2018). Other studies have evaluated how different reservation mechanisms of shared cars may be used to influence user behaviour (e.g., Wu et al. 2019 analyze how users respond to different reservation schemes adopted for tackling imbalances between supply and demand in free-floating carsharing).

A major topic of investigations has been represented by the development of methods for choosing where to locate charging stations for electric carsharing. Limiting our attention to some more recent works, we recall the studies: (Cheng et al., 2019), which proposes to combine statistical models with machine learning techniques not only to identify sites for deploying new charging stations, but also for adjusting the location of stations already deployed; (Chen et al., 2018), which analyzes the performance of regression models for evaluating how station features, city environment and transportation facilities affect carsharing services and proposes a method for deciding the location of stations; (Wang et al., 2019), which develops a four-step method that first estimates and distributes charging demand of electric vehicles in a city and then establishes the location of normal and fast charging stations for both private and shared vehicles; (Biondi et al., 2016), which proposes an optimization approach for establishing the costoptimal location and capacity of charging stations, using queueing theory for expressing the demand of recharge; finally, (Li et al., 2017) proposes a multi-criteria decision approach, including factors like travel purposes and 
distance from existing stations, for optimally locating carsharing stations and tests its performance on EVCARD, the electric carsharing system of Shanghai.

Another major topic of investigations has been represented by the problem of relocating vehicles, which can sensibly increase the profit of carsharing, as highlighted in the recent survey (Illgen and Höck, 2019) and in other works such as: 1) (Boyaci et al., 2017), which proposes a simulationoptimization approach for a station-based one-way carsharing system that provides for reservations and relocations; 2) (Bruglieri et al., 2018), which proposes a two-phase heuristic for solving a 3-objective carsharing relocation problem, considering cost minimization, fair distribution maximization and service level maximization; 3) (Zhao et al., 2018), which focuses on optimally managing vehicle rebalancing and staff relocation in a station-based one-way carsharing system, proposing an innovative algorithm combining Lagrangian relaxation and dynamic programming for its solution.

In the present work, we review some major city carsharing regulations, highlighting the importance of adopting reserved parking slots for carsharing vehicles. Also, we propose a mathematical optimization model and algorithm for establishing where to put reserved parking slots at disposal of carsharing users. The reserved parking slots are simple parking spaces meant to be spread around a city more pervasively of renting/charging stations. Their essential purpose is to speed up the parking phase of a rent, reducing the cruising time needed by a carsharing user for finding an empty slot.

Specifically, our major contributions are the following:

1. we provide an overview of the carsharing regulations of a number of major cities, particularly highlighting the importance of parking policies in making carsharing a success and discussing the Italian case in more detail;

2. given the crucial role of parking policies in carsharing, we characterize the optimization problem of a Local Government (for example the council of a municipality) that must choose which parking slots to rent to carsharing companies in a city, while finding an optimal balance between the interest of the population and those of the profit-oriented companies. To model this problem, we propose to adopt mathematical optimization techniques and we represent the problem as a Binary Linear Programming problem, which includes boolean variables to model the possibility of renting or not a cluster of parking slots. The purpose of this model, is to provide an easy-to-use mathematical tool for Local Governments, which can be easily adopted and tuned. 
To the best of our knowledge, such decision problem has never been considered in literature and addressed through optimization techniques as we do in the present paper. We thus remark that there is no related literature to review and compare with;

3. we prove that the considered optimization problem is NP-Hard and thus can prove difficult to solve even for state-of-the-art optimization software. To tackle the problem, we therefore propose a new matheuristic that combines a genetic algorithm with exact mathematical programming techniques adopted in large neighborhood searches;

4. we present the results of computational tests on realistic instances referring to the city of Rome. Such data are also defined on the basis of the experience gained within our collaborations with major carsharing companies active in Rome and with E-Go, a carsharing service launched at University Roma Tre with the support of the electric utility company Enel (see Carrese et al. 2017). They also take into account the current regulations of the City of Rome for carsharing (City of Rome - DGC136, 2016).

The remainder of the paper is organized as follows. In Sections 2, we overview city regulations, whereas in Section 3, we define the model for optimal parking slot renting. In Sections 4 and 5, we present the matheuristic and the results of computational tests, respectively. In Section 6, we discuss challenges related to the real-world adoption of the proposed optimization approach. Finally, in Section 7, we derive conclusions and discuss possible directions for future work.

\section{A review of local regulatory situations for carsharing}

As a major expression of SMOB, carsharing has widely spread especially in the USA and Europe, becoming one of the new important modes of urban transport (Pinna et al., 2017). Several studies pointed out that the use of privately-owned cars, though still very common, has experienced a significative decline in many countries since several years (see e.g., Millard-Ball and Schipper 2011; Newman and Kenworthy 2011). Furthermore, carsharing has gained a lot of popularity as a more sustainable way to reduce emissions of CO2 (Martin and Shaheen, 2011).

A good parking policy is indicated in literature as one of the most effective strategies that a local government can implement to stimulate carsharing (e.g., Rivasplata et al. 2013). A detailed study made in (Shaheen et al., 2010) highlights that in North America over 70 local governments 
and municipalities, such as San Francisco and Vancouver, have adopted specific policies to favour the parking of carsharing, also including the reservation of parking slots for shared cars. This study also reports that a survey among the San Francisco residents revealed that just 20\% of the people were against the reservation of slots for carsharing. Recently, also the New York City Council has approved legislation for a pilot study aimed at evaluating the reservation of parking slots to carsharing companies (see New York City Council 2015).

Another very interesting study that has investigated how local governments can support and stimulate carsharing, in particular by reserving parking slots is (Dowling and Kent, 2015). The authors focused on the case of Sydney, which in 2013 offered 1000 shared cars managed by various companies, leading to an estimated benefit of more than 300 millions of dollars for carsharing users. The study highlights that the carsharing was not taken into consideration in the regional transport plans, but was instead considered at a local level, in the regulations of Sidney city and in that of 6 out of 8 the districts where carsharing operated.

In 2016, the City of Sydney has approved new regulations for managing on-street carsharing parking slots with the aim of exploiting them more efficiently - see (City of Sydney, 2016). A first objective of these regulations is to define the requirements and obligations of companies that apply for reserved carsharing parking slots: for example, they require to deploy vehicles that emit less than $175 \mathrm{~g} / \mathrm{km}$ of $\mathrm{CO} 2$ and to produce a report about the use of each vehicle each month. A second objective is to clearly state the rules by which a company may get parking slots: the rent must obtain a preventive approval from the residents and retailers of the area and from local committees for bicycle and pedestrian mobility. The number and position of the parking slots are defined on the basis of an evaluation of the potential demand for carsharing and on the basis of the district features: if in a district less than $3.5 \%$ of all the slots are reserved to carsharing, then a company already renting slots may request at most 4 slots, if it can prove that the 3 slots that are closest to those requested have been used at least 18 times in a month; for districts exceeding the threshold of 3.5\%, the conditions are more stringent. In order to favour competition, a company without rented slots can rent without restrictions at most 3 slots in a district with up to 900 total slots or at most 6 in a district with more than 900 slots. Always in the metropolitan area of Sydney, specifically in the Municipality of Ashfield, the local government has approved regulations specific for carsharing (Municipality of Ashfield, 2010), which were aimed at deeply involving the citizens in the process of assignment of parking slots. 
Assigning slots to carsharing in an area required the approval of $75 \%$ of the residents. Furthermore, the rules favoured the location of slots close to parks and retailers. Last but not least, they provided for making pay the cost of realization of the reserved slots to the carsharing companies, which every year must submit a report on the usage of the slots.

In 2011, the Canadian city of Calgary approved regulations aimed at favouring carsharing specifically impacting on parking policy (City of Calgary, 2011). In particular, the carsharing companies are obliged to relocate their vehicles so that the reserved slots have a car available for a minimum number of hours everyday. Also, each company may receive at most $3 \%$ of the total number of slots available in any area identified as commercial. Finally, it is interesting to cite the case of the city of Vancouver, where the price for renting parking slots differs depending upon the district (City of Vancouver, 2016): more attractive districts in terms of business activities are associated with higher prices.

Concerning Italy, urban carsharing is currently active in 29 cities and, according to a 2016 survey, was counting 5764 vehicles. Concentrating the attention on three of the largest cities (Milan, Rome and Turin), the first very interesting observation to be made is that, though carsharing was introduced in these cities many years ago, it has been interested by a limited regulation and emission of policies, particularly those on parking, aimed at improving its effectiveness and efficiency. The city of Milan offers the most advanced urban carsharing system in Italy, with several companies operating in the city. In 2013, new regulations were introduced to allow the shared cars to access and park for free in central restricted traffic zones and to park in slots restricted to residents. The regulations also state that a carsharing company must pay a yearly fixed price of 1100.00 euros for operating a vehicle in the city and access the benefits stated above. The more recent regulations (City of Milan, 2016) have introduced the obligation for the carsharing company to regularly update their fleet, imposing that a vehicle must be replaced once reached 4 years of service or 100,000 km.

The city of Rome has also been interested by regulations, similar to those of Milan: first regulations from 2004 allowed free access and parking in central restricted traffic zones; then, in 2010, it has been imposed that shared vehicles must be at most 3 years old. Finally, in 2016, further regulations - e.g., (City of Rome - DGC136, 2016) - have been aimed at strengthening the penetration of electric vehicles, establishing the realization of additional charging stations in central zones of the city.

In Turin, the regulations (City of Turin, 2015) not only allow the free access and parking in central restricted traffic zones, but also grant the right 
to drive on fast lanes reserved to bus and taxi services. A distinctive feature of these regulations is that they have been especially aimed at containing the air pollution, by imposing that the fleet of each carsharing company must contain at least $30 \%$ of low-emission natural-powered vehicles (e.g., bi-fuel methane-gasoline) and at least 10 electric vehicles. Furthermore, the regulations provide for that recharge stations of the company must be open also to private electric vehicles and that each company must cover with service an area of at least 40 square kilometers.

On the basis of the overview provided in this section, it can be seen that parking policies constitute an important element of regulations adopted to encourage the development and penetration of carsharing in cities. We can distinguish two major parking solutions: 1) assigning specific parking slots on the basis of a request made by a carsharing company; 2) renting a set of slots to all companies without distinction (i.e., no slot is reserved to a specific company). The chosen solution must be then accompanied by other decisions, such as: 1) the total number of slots made available in the city and in each zone; 2) the maximum number of slot reserved to each company; 3) the location of the slots. Furthermore, a parking policy should also fix the price that a company must pay to gain the right of using a slot. This price could be differentiated depending on the attractiveness of the area: commercial and business districts could be more attractive than residential districts and thus be associated with higher prices; also, larger groups of contiguous parking slots should be associated with higher prices, given the higher chances of attract carsharing vehicles and thus increasing availability for the users.

In order to guarantee a full application of the policy, the local governments must keep watch on the rented slots, timely sanctioning abuses and adopting ad-hoc fines for sanctioning people parking their private cars in reserved slots. We note that this aspect is not so trivial: the first experiments of carsharing directly managed by the City of Rome also failed because the reserved slots were often occupied by private cars, which counted on the very low chance of being fined by the local police. As found in several policies, it is also important that the local governments provides for obtaining regular reports about the conditions of each carsharing company, in particular defining some key performance indicators that allow to clearly evaluate the impact of carsharing and of parking slot reservation on the administered territory. Last but not least, the reservation of parking slots to carsharing should be subordinated to a positive advice of the local residents, as provided in the policies of several cities.

As it is evident, the problem that the local governments face when de- 
ciding how to put parking slots at disposal of carsharing companies presents a lot of factors to be taken into account. This leads to a complex decision problem that typically pursues the maximization of a measure expressing the benefit for the city and the population to adopt carsharing and specific parking policies. This is actually an optimization problem where the local government wants to take the best decision on the basis of its interest. In the next section, we investigate the possibility of modelling and solving such optimization problem through a mathematical optimization approach.

\section{An optimization model for car slot renting}

We consider the problem of the Local Government (LG) of a city that needs to decide which parking slots must be reserved and rented to carsharing companies that are active in its territory. The overall aim is to favour the diffusion of such services among the population, in order to improve the penetration of smart mobility and thus traffic conditions. We propose to tackle such problem by means of Mathematical Programming techniques (Bertsimas and Tsitsiklis, 1997): we first translate the problem faced by the LG into mathematical terms, deriving a suitable mathematical optimization model, and then propose a matheuristic algorithm for it solution. By adopting a Mathematical Programming approach, we allow to find a solution that grants the best performance according to an objective function that may includes key performance indicators.

We recall here some fundamentals of mathematical optimization, referring the reader to the book (Bertsimas and Tsitsiklis, 1997) for a more exhaustive and detailed introduction. An optimization model, also commonly called mathematical programming problem, is a mathematical model that is made up of three major components: 1) a set of decision variables, which model the choices that the decision maker can take (for example, if we refer to the parking slot renting problem that we consider in this paper, a variable models whether the LG does or does not rent a slot); 2) a set of feasibility constraints that model limitations on the value that can be assigned to the variables (for example, the LG cannot rent more than a fixed number of parking slots); 3) an objective function, which guides the search for the best solution of the problem, evaluating how good is a complete assignment of values to the variables.

We can express more formally some basic mathematical programming 
concepts by referring to the following problem denoted by MP:

$$
\begin{aligned}
\max & c^{T} x \\
& A x \geq b \\
& x \in\{0,1\}^{n}
\end{aligned}
$$

where $c \in \mathbb{R}^{n}, A \in \mathbb{R}^{m \times n}$, and $b \in \mathbb{R}^{m}$. We remark that the problem MP we consider is a Binary Linear Programming problem, since it only includes binary decision variables, assuming either 0 or 1 values, and linear constraints and objective function.

If we assign a value in $\{0,1\}$ to each decision variable $x_{j}, \forall j=1,2, \ldots, n$ of MP, we obtain a solution. A solution is either feasible, if it satisfies all the constraints (2), or infeasible, if it violates at least one of the constraints (2). If we denote by $\mathcal{F}$ the set of all feasible solutions, a feasible solution $x^{*} \in \mathcal{F}$ is said optimal if it offers the best objective value among all the feasible solutions, i.e. it maximizes the objective function (1) (formally, $\left.c^{T} x^{*} \geq c^{T} x, \forall x \in \mathcal{F}\right)$.

\subsection{System elements}

The optimization problem faced by the LG can be described as follows (we anticipate that in Figure 1, we provide a small example aimed at clarifying the notation introduced in the remainder of this section). The LG administers a city made up of a set of districts denoted by $D$. Each district $d \in D$ includes a set of subdistricts, denoted by $S(d)$. In each subdistrict $s \in S(d)$ with $d \in D$, the LG has identified a number of parking slot clusters available for renting to carsharing companies: a parking slot cluster (or briefly, cluster) is a set of parking slots that is reserved for parking carsharing cars. Formally, for each district $d \in D$ and subdistrict $s \in S(d)$, we denote by $C(s, d)$ the set of clusters available in $s$. For each cluster $c \in C(s, d)$, we denote by $n_{c}$ the number of parking slots composing the cluster. A cluster must be rented as a whole, i.e. it is not possible to just rent a part of its slots. As in real-world studies, we assume that the LG has identified a profit measure $\pi_{c}$ that quantifies the benefits of renting a cluster $c$ on the basis of preliminary studies (see, for example, the regulation of the City of Rome - DGC136 2016). Such measure may take into account several distinct factors, such as the revenue obtained renting the cluster, the cost associated with maintaining the cluster, the economical benefits of having carsharing services in an area (e.g., financial, environmental and social).

In line with policies overviewed in the previous section, we strongly believe that the renting of parking slots should be coordinated with local residents. To this end, we consider very important to include a limit on the 


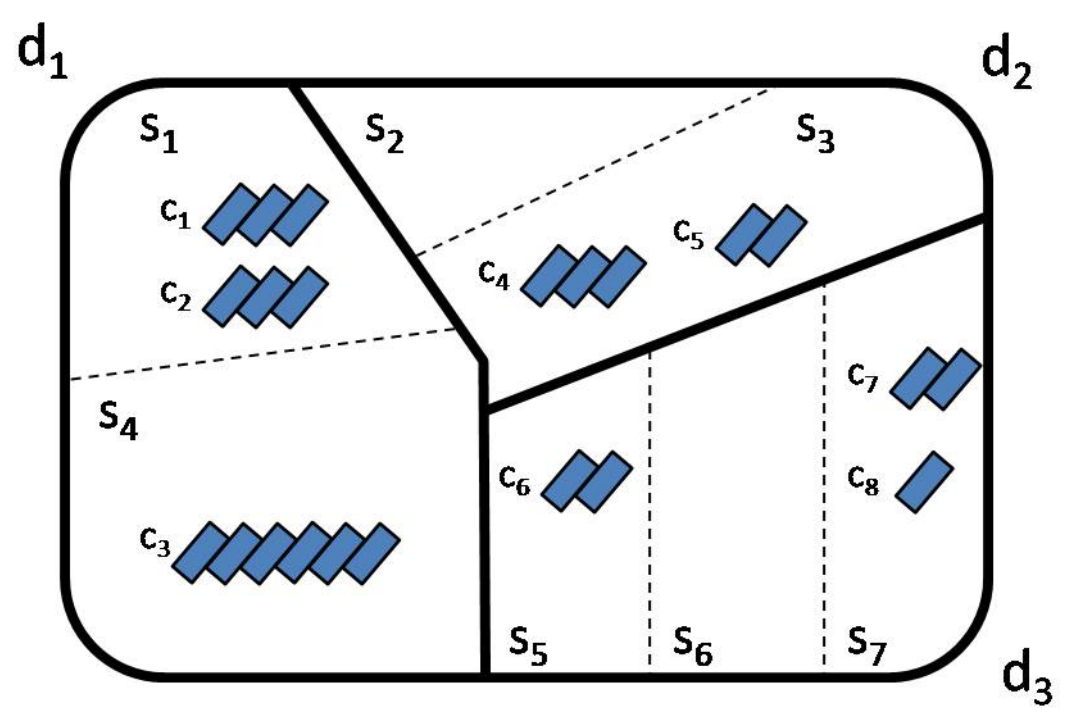

Figure 1: An example of target area partitioned into districts and subdistricts, containing parking slot clusters. The districts are separated by thick continuous lines, whereas the subdistricts of a district are separated by dotted thinner lines. Parking slots clusters are represented by groups of small rectangles placed side by side and each rectangle visualizes one slot. Referring to the notation introduced in Subsection 3.1, this example of target area contains three districts, so $D=\left\{d_{1}, d_{2}, d_{3}\right\}$. The subdistricts of each districts are: $S\left(d_{1}\right)=\left\{s_{1}, s_{4}\right\}, \quad S\left(d_{2}\right)=\left\{s_{2}, s_{3}\right\}$ and $S\left(d_{3}\right)=\left\{s_{5}, s_{6}, s_{7}\right\}$. Concerning the parking slot clusters, we note that subdistricts $s_{2}$ and $s_{6}$ do not contain clusters available for renting inside them (so, $C\left(s_{2}, d_{2}\right)=C\left(s_{6}, d_{3}\right)=\emptyset$ ). Instead, all the other subdistricts contains at least one cluster (for example, $s_{1}$ has 2 clusters, $C\left(s_{1}, d_{1}\right)=\left\{c_{1}, c_{2}\right\}$, both containing 3 parking slots (i.e., $n_{c_{1}}=n_{c_{2}}=3$ ), whereas $s_{4}$ contains only cluster $c_{3}$ including 6 slots (i.e, $C\left(s_{4}, d_{1}\right)=\left\{c_{3}\right\}$, with $\left.n_{c_{3}}=6\right)$. 
total number of parking slots that can be rented in each district. This is done for avoiding to arouse widespread discontent among local residents, which typically wants to have a consistent fraction of the parking slots in a district to be (freely) available to car owners. For example, the number of rented slots could be required to not exceed a fraction of the total number of parking slots available in the district. Furthermore, it is also important to include a minimum number of slots that must be rented, to favour the diffusion of carsharing; this could reflect the dimension of the fleet maintained by the companies. For each sub-district $s \in S(d)$ with $d \in D$, we denote such lower and upper limits on the number of rented parking slots by $\eta_{s}^{\min }$ and $\eta_{s}^{\max }$, respectively. Furthermore, we assume that the LG also wants to include a lower and upper limit $\gamma_{s}^{\min }, \gamma_{s}^{\max }$ on the number of clusters that may be rented in each subdistrict.

Another important aspect that we want to model is the possibility for the LG to classify the clusters per types and to include a minimum and maximum number of clusters for each type in each district. For example, clusters could be distinguished per type by the number of parking of slots that they include. On the basis of our direct experience with the creation of a new carsharing service in Rome, we expect that an LG wants to have a good balance between clusters of bigger and smaller dimensions. Another example of type distinction is that between shopping, business and residential clusters, depending in which zone of a district they are located. From a modelling point of view, we introduce a set $T$ to denote the cluster types and we denote by $t(c) \in T$ the type of a cluster $c$. For each district $d \in D$, we denote by $\tau_{d t}^{\min }$ and $\tau_{d t}^{\max }$ the minimum and maximum number of clusters of type $t$ allowed in district $d$. In Figure 1, we provide an example to clarify the notation introduced.

\subsection{Modelling the optimization problem}

After having introduced all the relevant system elements and the related notation, we can state the optimal decision problem that the LG faces as follows.

Optimal Carsharing Parking Slot Renting Problem (Opt-ParkRent): given the set of districts $D$, the corresponding sets of subdistricts $S(d) \forall d \in D$ and sets of parking slot clusters $C(s, d) \forall d \in D, s \in S(d)$, the profit $\pi_{c}$ and number of slots $n_{c}$ of each cluster $c \in C(s, d)$, the upper and lower bounds $\eta_{s}^{\min }, \eta_{s}^{\max }, \gamma_{s}^{\min }, \gamma_{s}^{\max }, \tau_{d t}^{\min }$ and $\tau_{d t}^{\max }$ on the number of rentable slots, rentable clusters and rentable cluster types, Opt-ParkRent consists of choosing which clusters to rent to carsharing services, so that all lower and 
upper bounds on rentable slots, rentable clusters and rentable cluster types are satisfied and the total profit is maximized.

We model the optimization problem Opt-ParkRent as a Binary Linear Programming problem. In order to model the decision of renting or not a parking slot cluster, we introduce a binary decision variable $x_{d s c} \in\{0,1\}$ for each district $d \in D$, subdistrict $s \in S(d)$ and cluster $c \in C(s, d)$, defined as follows:

$$
x_{d s c}= \begin{cases}1 & \text { if cluster } c \text { in subdistrict } s \text { of district } d \text { is rented } \\ 0 & \text { otherwise }\end{cases}
$$

These decision variables are employed in the following constraints defining the set of feasible solutions of the optimization problem. First, we need a set of constraints to express that, for each subdistrict, the limits on the number of rented parking slots cannot be exceeded:

$$
\eta_{s}^{\min } \leq \sum_{c \in C(s, d)} n_{c} \cdot x_{d s c} \leq \eta_{s}^{\max } \quad \forall d \in D, s \in S(d)
$$

We remark that here the decision variable is multiplied by the number $n_{c}$ of slots in a cluster.

Then, we must express the limits on the number of clusters that can be rented in each subdistrict:

$$
\gamma_{s}^{\min } \leq \sum_{c \in C(s, d)} x_{d s c} \leq \gamma_{s}^{\max } \quad \forall d \in D, s \in S(d)
$$

Finally, we need constraints to express the limits on the number of cluster types that can be rented in each district:

$$
\tau_{d t}^{\min } \leq \sum_{s \in S(d)} \sum_{c \in C(s, d): t(c)=t} x_{d s c} \leq \tau_{d t}^{\max } \quad \forall d \in D, t \in T
$$

We note that in these constraints the two summations involve the decision variables of all the clusters located in sub-districts of the district $d$ that are of cluster type $t$.

The objective is to maximize the total profit, expressed as the summation of the decision variables over all districts, subdistricts and clusters:

$$
\max \sum_{d \in D} \sum_{s \in S(d)} \sum_{c \in C(s, d)} \pi_{c} \cdot x_{d s c}
$$


By joining (4) - (8), we obtain the overall Binary Linear Programming problem presented below, denoted by BLP-PS, which we use as basis to model and solve the problem of renting parking clusters Opt-ParkRent.

$$
\begin{array}{rlrl}
\max & \sum_{d \in D} \sum_{s \in S(d)} \sum_{c \in C(s, d)} \pi_{c} \cdot x_{d s c} & & \text { (BLP-PS) } \\
\eta_{s}^{\min } & \leq \sum_{c \in C(s, d)} n_{c} \cdot x_{d s c} \leq \eta_{s}^{\max } & & \forall d \in D, s \in S(d) \\
\gamma_{s}^{\min } & \leq \sum_{c \in C(s, d)} x_{d s c} \leq \gamma_{s}^{\max } & & \forall d \in D, s \in S(d) \\
\tau_{d t}^{\min } & \leq \sum_{s \in S(d)} \sum_{c \in C(s, d): t(c)=t} x_{d s c} \leq \tau_{d t}^{\max } & & \forall d \in D, t \in T \\
x_{d s c} & \in\{0,1\} & d \in D, s \in S(d), c \in C(s, d)
\end{array}
$$

Concerning the computational complexity of our problem Opt-ParkRent associated with parking slot optimization and modelled as BLP-PS, we now show that it is NP-Hard. To this end, we state and prove the following proposition.

Proposition 1. Opt-ParkRent is NP-Hard.

PROOF. In order to prove the result, we refer to the optimization model BLP-PS that we proposed to model Opt-ParkRent. We can first note that all the feasibility constraints (5), (6) and (7) present the following general form:

$$
L B_{i} \leq \sum_{j \in J} a_{i j} \cdot x_{j} \leq U B_{i}
$$

Here, $i$ is the index of a generic constraint (we denote by $I$ the set of constraint indices) and $j$ is the index of a generic decision variable (we denote by $J$ the set of variable indices). Each constraint contains a summation over all binary decision variables $x_{j}$ multiplied by suitable non-negative coefficients (in particular, we note that we have $a_{i j}=0$ when $x_{j}$ is not included in a constraint, $a_{i}=1$ when $x_{i}$ appears in constraints (6) and (7) and $a_{i}=n$, with $n$ representing the number of parking slots associated with $x_{i}$ when $x_{i}$ appears in a constraint (5)). Each summation is then bounded below by a 
value $L B_{i}>0$ and above by $U B_{i}>0$. The overall problem then reads as:

$$
\begin{array}{cl}
\max & \sum_{j \in J} \pi_{j} \cdot x_{j} \\
L B_{i} \leq \sum_{j \in J} a_{i j} \cdot x_{j} \leq U B_{i} & \forall i \in I \\
x_{j} \in\{0,1\} & \forall j \in J
\end{array}
$$

The previous problem constitutes a multidimensional generalization of the knapsack problem with minimum filling constraints, introduced and proved to be NP-Hard in $(\mathrm{Xu}, 2011)$. As a consequence, also the problem OptParkRent is NP-Hard.

\section{A Genetic-based Matheuristic for solving BLP-PS}

Because of its NP-Hard nature, the parking slot optimization problem Opt-ParkRent, modelled by BLP-PS, can be hard to solve even for state-ofthe-art optimization solvers, such as IBM ILOG CPLEX. We therefore propose to solve BLP-PS by a matheuristic that combines a genetic algorithm with solution generation and improvement phases based on the execution of suitable large neighborhood searches. Matheuristics are a relatively recent trend in metaheuristic methods that tries to better exploit the potentialities of exact mathematical optimization techniques through hybridization with heuristic methods (see e.g., Maniezzo et al. 2009). Concerning the use of large neighborhood searches, we stress that we rely on exact searches, namely the search is not a simple local search based on heuristic rules of exploration, but a large exploration that is formulated as an optimization problem solved exactly by a state-of-the-art solver (see e.g., Blum et al. 2011 for an introduction). The rationale at the basis of exact searches is that, while a state-of-the-art solver may find big difficulties in solving a full problem, it may instead be able to effectively and efficiently solve suitable subproblems obtained from it and associated with the large neighborhood searches.

Genetic Algorithms (GAs) are well-known heuristic algorithms inspired from the evolution dynamics of a population of individuals. For an exhaustive introduction to theory and applications of GAs, we refer the reader to (Golberg, 1988; Karakatic et al., 2015; Srinivas et al., 1994). Focusing on its essential features, a GA manages a population of individuals, in which each individual represents a feasible solution for the optimization problem at hand. The chromosome of an individual specifies the value that is assumed 
1: Creation of the initial population

2: while an arrest condition is not satisfied do

3: $\quad$ Selection of individuals who generate the offspring

4: $\quad$ Generation of the offspring by crossover

5: Mutation of part of the population

6: Death of part of the population

7: end while

8: Improvement by Exact Large Neighborhood Search

451

detail the features of all the steps of the algorithm.

\subsection{Characteristics of the GA population}

\subsubsection{Representation of the individuals and fitness function}

In the case of our problem, a natural modelling choice within the GAbased matheuristic is to let coincide the chromosome of an individual with the binary vector $x$ specifying the value of each variable $x_{d s c}$ with $d \in D, s \in$ $S(d), c \in C(s, d)$ included in BLP-PS.

It is also natural to adopt the objective function (8) of BLP-PS as fitness function. For a given individual associated with a chromosome $x$, the fitness of the corresponding individual is thus equal to the revenue of the rented 作 etail, the general structure of the GA that we use as basis for 
clusters, namely:

$$
\pi(x)=\sum_{d \in D} \sum_{s \in S(d)} \sum_{c \in C(s, d)} \pi_{c} \cdot x_{d s c}
$$

\subsubsection{Initial population}

In order to define the individuals composing the initial population, we rely on both a deterministic and a probabilistic individual generation procedures.

The deterministic generation is based on a procedure that follows the principles of RINS (Relaxation Induced Neighborhood Search) (Danna et al., 2005), a well-known heuristic for Mixed Integer Linear Programming. Specifically, let BLP-PS ${ }^{R L X}$ be a linear relaxation of BLP-PS (i.e., a version of BLP-PS obtained by substituting the integrality requirement $x_{d s c} \in\{0,1\}$ on each decision variable with its continuous relaxation $0 \leq x_{d s c} \leq 1$ ) and let $x^{\text {RLX }}$ be its optimal solution. The optimal solution $x^{\mathrm{RLX}}$ can be used as basis to fix a-priori the value of some variables in BLP-PS, thus obtaining a smaller and easier-to-solve problem that can be tackled by a state-of-the solver. We denote by BLP-PS ${ }^{\text {FIX-RINS }}$ the problem where some variables have their value fixed a-priori and the strategy for fixing is the following:

$$
\begin{array}{llll}
\text { IF } & x_{d s c}^{\mathrm{RLX}} \leq 0+\epsilon & \text { THEN } & x_{d s c}=0 \\
\text { IF } & x_{d s c}^{\mathrm{RLX}} \geq 1-\epsilon & \text { THEN } & x_{d s c}=1
\end{array}
$$

with $\epsilon>0$. In other words, the rule provides for fixing to either 0 or 1 a variable that in $x_{d s c}^{\mathrm{RLX}}$ is sufficiently close to 0 or 1 . Indeed, such closeness can be considered a good indication that the variable should be fixed to that value in a solution of good quality (see Danna et al. 2005 for an exhaustive discussion of the principles of the RINS algorithm). The problem BLP-PS ${ }^{\text {FIX-RINS }}$ that we obtain by the RINS-fixing procedure is passed to a state-of-the-art solver and we add all the feasible solutions found during the solution procedure to the initial population of the GA algorithm.

We also generate solutions through a partial probabilistic variable fixing procedure that uses again the optimal solution $x^{\mathrm{RLX}}$ of the linear relaxation, but in a different way. To this end, we can first note that a fractional optimal value $x_{j}^{\mathrm{RLX}}$ can be interpreted as the probability of fixing to 1 the corresponding variable $x_{j}$ in a good feasible solution (we refer the reader to the book by Motwani and Raghavan (1995) and to the papers (Maniezzo, 1999; D'Andreagiovanni et al., 2015; D'Andreagiovanni and Nardin, 2015) for a discussion about the interpretation of fractional binary solutions as 
probability in randomized rounding algorithms in a general and in a metaheuristic context). We thus create a number of solutions using $x_{j}^{\mathrm{RLX}}$ as probabilities and confronting them with randomly generated numbers, similarly to Ant Colony Optimization procedures illustrated in (Maniezzo, 1999; D'Andreagiovanni et al., 2015; D'Andreagiovanni and Nardin, 2015). This probabilistic generation procedure is operated for a part of all the variables and the resulting partial fixing is passed to a state-of-the-art solver for solving the resulting problem of reduced size (again, we add all the feasible solutions found during the solution procedure to the initial population of the GA algorithm).

\subsection{Evolution of the population}

\subsubsection{Selection}

In order to select the individuals that combine their chromosomes for generating the new population, we execute a tournament selection: if $\mathcal{P}$ is the current population of individuals, as first step we define $k>0$ subgroups of individuals through random selection of a number $\lfloor\alpha \cdot|\mathcal{P}|\rfloor$ of individuals from $\mathcal{P}$, with $\alpha \in(0,1)$. Then we select the $m<\lfloor\alpha \cdot|\mathcal{P}|\rfloor$ individuals that offer the best fitness function value in each group. We extract individuals from this restricted pool for giving raise to the new generation by means of crossover.

\subsubsection{Crossover}

Individuals belonging to the restricted pool defined in the previous step are randomly paired so to constitute $\lfloor k \cdot m / 2\rfloor$ couples. From each couple, we generate one offspring through crossover of chromosomes. Specifically, given a couple of individuals (the parents) associated with two solution vectors $x^{1}, x^{2}$ of the model BLP-PS, the crossover mixes the values of variables in the same position of the parents, so to give birth to one offspring $x^{\text {off }}$, hopefully associated with higher fitness value.

Specifically, $x^{\text {off }}$ is defined according to two rules:

1. if the parents present the same binary value in a position $j$, then the offspring inherits this value in the same position $j$ (i.e., if $x_{j}^{1}=x_{j}^{2}$ then $\left.x_{j}^{\text {off }}=x_{j}^{1}\right)$;

2. if the parents have distinct binary values in a position $j$ (i.e., $x_{j}^{1} \neq x_{j}^{2}$ ), then the variable $x_{j}^{\text {off }}$ is not fixed and its value is decided by running an optimization solver. Specifically, let BLP-PS ${ }^{\text {FIX }}$ be the subproblem of BLP-PS obtained by fixing a subset of variables according to the previous rule 1 . Then we set as offspring the best feasible solution found 
by a state-of-the-art solver used to solve BLP-PS ${ }^{\text {FIX }}$ within a time limit (so, again we exploit the solver to tackle a suitable subproblem of the complete hard-to-solve problem BLP-PS).

The rationale at the basis of these two crossover rules is that two solutions presenting the same valorization of a variable constitute a good indication that such valorization should be kept in the offspring, whereas for deciding how to valorize variables with conflicting values in the parent solutions, we exploit the solver to tackle a subproblem of the complete hard-to-solve problem BLP-PS.

\subsubsection{Mutation}

After having created the new generation of individuals, we provide for trying to obtain diversified solutions and possibly escaping from local optima by applying the mutation technique: we randomly choose a number of individuals $\lfloor\gamma \cdot|\mathcal{P}|\rfloor$ with $0<\gamma<1$ and we randomly choose a number of positions in the chromosome whose binary value is inverted. If this leads to make the solution associated with the individual infeasible, then the individual is discarded and is included in the count of the death process described below.

\subsubsection{Death}

After the execution of crossover and mutation operations, we attempt at removing the weakest individuals in the population, mimicking a process of death. To this end, $\lfloor k \cdot m / 2\rfloor$ individuals presenting the lowest fitness value are discarded.

\subsection{Improvement by Exact Large Neighborhood Search}

Once that the GA algorithm has been run iteration after iteration, reaching the time limit for its execution, we use the best feasible solution found as basis for implementing an exact large neighborhood search. Specifically, let $x^{\text {BEST }}$ be such best feasible solution, we try to improve it searching a better solution in the neighborhood that includes all the feasible solutions obtainable by inverting the binary value of at most $\Gamma>0$ entries of $x^{\mathrm{BEST}}$. Since we implement an exact search, as explained in previous subsections, we need to formulate the search as an optimization problem solved by a stateof-the-art-solver. We can accomplish this by adding the following hamming distance constraint to BLP-PS:

$$
\sum_{j \in J: x_{j}^{\mathrm{BEST}}=0} x_{j}+\sum_{j \in J: x_{j}^{\mathrm{BEST}}=1}\left(1-x_{j}\right) \leq \Gamma \quad(H D)
$$



PS is presented in Algorithm 2. ber must be not larger than $\Gamma$. art-solver with a time limit.

Indeed, this constraint counts the number of binary variables that change values with respect to the best feasible solution found $x^{\mathrm{BEST}}$ and such num-

We denote by BLP-PS ${ }^{\mathrm{HD}}$ the modified problem BLP-PS that includes the hamming distance constraint (HD), which we then solve by a state-of-the-

An overview of the phases of the matheuristic algorithm for solving BLP-

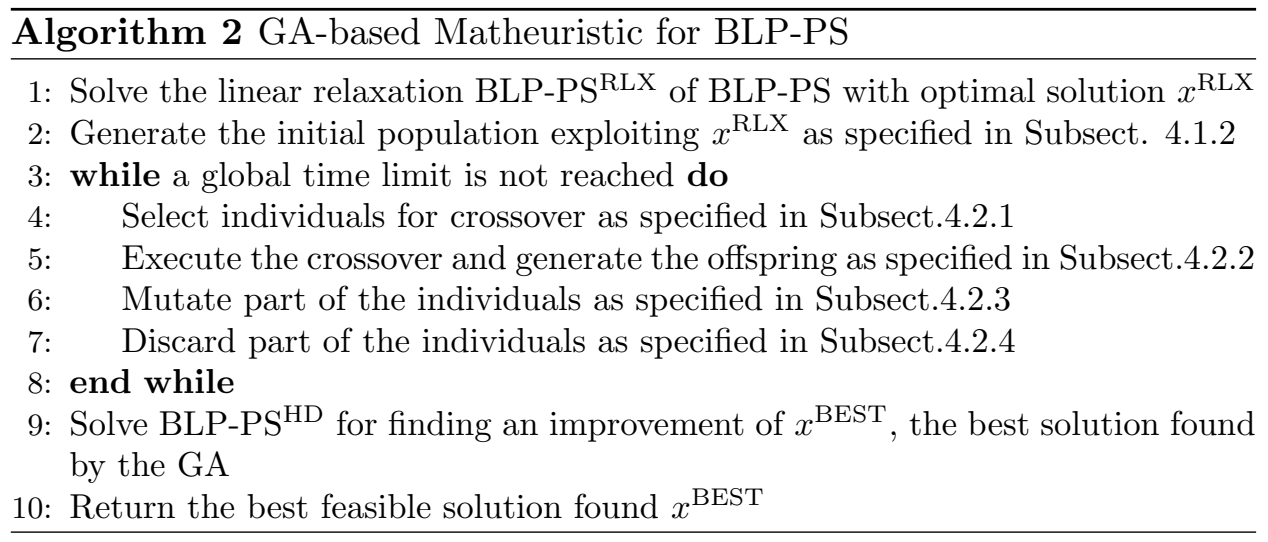

\section{Computational results}

We employed the optimization model BLP-PS and the GA-based Matheuristic defined in the previous sections to solve Opt-ParkRent - the Optimal Carsharing Parking Slot Renting Problem introduced in Section 3.2 - using realistic data referring to the Italian city of Rome. These data were defined on the basis of the specific regulations of the city (City of Rome DGC136, 2016) and of our experience in collaborating with professionals of major carsharing companies active in Rome and with E-Go, a carsharing service launched at University Roma Tre with the support of the electric utility company Enel (see Carrese et al. 2017).

Inside each subdistrict, we identified a number of parking slot clusters, on the basis of the features of the subdistrict (size, road configuration, importance in the Roman urban mobility system). Concerning the cluster types, we identified 5 types on the basis of the number of parking slots included in a cluster. Each cluster presents a number of slots ranging from 1 to 12 , with bigger clusters located close to important landmarks (for example, train stations). The lower and upper bound on the number of slots 
rentable in each subdistrict, those on the number of clusters rentable in each subdistrict and those on the number of types rentable for each type in each district (i.e., $\eta_{s}^{\min }, \eta_{s}^{\max }, \gamma_{s}^{\min }, \gamma_{s}^{\max }, \tau_{d t}^{\min }$ and $\tau_{d t}^{\max }$ ), are defined considering the features of the resident population, business activities, available public transportation and urban fabric.

The matheuristic solution algorithm was run on a $2.70 \mathrm{GHz}$ Windows machine with 8 GB of RAM and using IBM ILOG CPLEX 12.5 as software for solving the optimization subproblems (CPLEX, 2020). The code was written in $\mathrm{C} / \mathrm{C}++$ and interfaced with CPLEX through Concert Technology.

The matheuristic ran with a total time limit of 3600 seconds: 3000 seconds were granted to the construction phase, while 600 seconds were granted to the improvement phase. The parameters of the GA algorithm were the following: the tournament selection involves $k=10$ groups, in which a fraction $\alpha=0.1$ of individuals of the population $\mathcal{P}$ are included; the crossover is operated on the $m=10$ individuals presenting the highest fitness value in each group. After having created new individuals, a fraction $\gamma=0.05$ of the population undergoes mutation. Finally, we set $\epsilon=0.1$ in the RINS search and $\Gamma$ equal to $10 \%$ of the total number of decision variables in the hamming distance constraint employed in the exact search for improved solutions.

As first test, we considered a quite small instance, in terms of number of clusters, made up of 5 districts and including 27 subdistricts in total, where we identified 191 potentially rentable clusters. Each subdistrict contains from 2 to 22 clusters, whose number of slots ranges from 1 to 10 (bigger clusters are located close to important landmarks, for example, train stations). The results associated with the optimal solution of the problem are visualized in Figures $2 \mathrm{a}$ and $2 \mathrm{~b}$. The solution specifies the clusters that must be rented in each subdistricts, in order to maximize the total profit while respecting renting constraints.

As a comment to the results, we can first note that, if the problem contained just one single constraint imposing a maximum number of slots over all the subdistricts, then we could adopt a simple and fast heuristic solution approach consisting of: 1) computing the profit per parking slot of each cluster; 2) sorting the clusters from the highest to the lowest in terms of such profit; 3 ) select one by one the clusters, following the sorting order, until the maximum number of rentable slots is exceeded. However, this simple solution approach would possibly lead to a solution of quite low quality offering an unfair distribution of the clusters over the territory. To avoid this, the additional constraints that limit the number of slots, the number of clusters and the number of types of clusters are needed. This results into a more complex structure of the problem, where the connections 


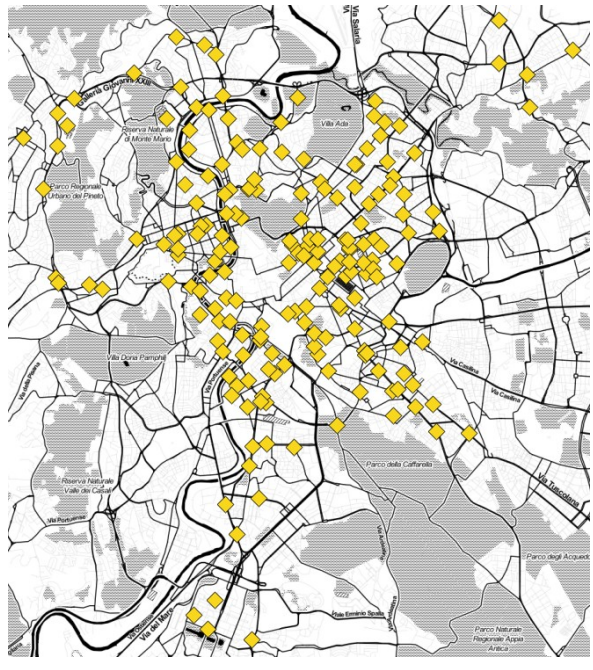

(a) Candidate clusters

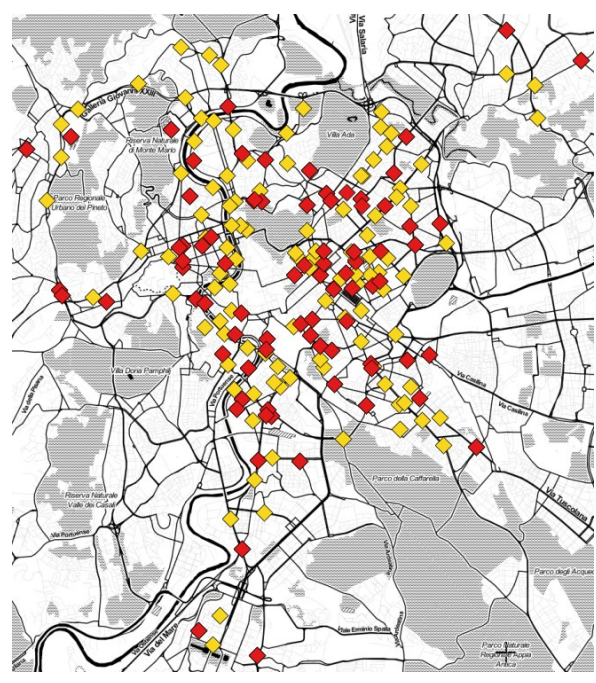

(b) Optimal selection (in red) of clusters

Figure 2: Parking clusters for the City of Rome

between districts, subdistricts and cluster types discourage the application of the previously cited simple heuristic solution and requires instead the application of a more sophisticated solution algorithm like our matheuristic.

Analyzing the optimal solution of this small instance and putting it into relation with the input data of the optimization problem, we can note that there is a tendency to push the number of rented clusters to its imposed minimum $\eta_{s}^{\min }$ in less profitable subdistricts. In contrast, the number of activated clusters tends to be closer to the allowed maximum $\eta_{s}^{\max }$ in more attractive subdistricts, where clusters containing a higher number of parking slots are present. Going more into details, in one of the largest downtown subdistrict, which hosts important business and administrative activities and is close to important public transportation nodes, it is activated one of the largest clusters plus a combination of well-spaced clusters with 5 and 7 slots. As it can be observed in the figure, the presence of the constraints expressing limitations on the rentable clusters allow to have a fair distribution of the rented clusters over all the districts and subdistricts, thus contributing to pursue a fundamental objective of the LG.

After this test, we considered an instance of larger size, containing a sensibly higher number of parking slot clusters, namely 500 in total, and we studied the effect of varying the upper bounds $\eta_{s}^{\max }$ on the total number of rentable slots and $\gamma_{s}^{\max }$ on the total number of rentable clusters. The 
Table 1: Results for varying upper bounds $\gamma_{s}^{\max }$ and $\eta_{s}^{\max }$ for a 500 cluster instance. For each combination of the two bounds, the table reports two values: 1) the number of activated clusters; 2) the total profit associated with the solution (in EUR).

\begin{tabular}{|c|c|c|c|c|c|c|c|c|c|}
\hline & & \multicolumn{8}{|c|}{$\gamma^{\max \%}$} \\
\hline & & & 5 & & 10 & & 15 & & 20 \\
\hline \multirow{6}{*}{$\eta^{\max \%}$} & 5 & 28 & 802935 & 31 & 815747 & 31 & 815747 & 31 & 815747 \\
\hline & 10 & 32 & 1516350 & 43 & 1605688 & 46 & 1607896 & 46 & 1607896 \\
\hline & 15 & 32 & 1944942 & 53 & 2300368 & 62 & 2351909 & 65 & 2352546 \\
\hline & 20 & 32 & 2065162 & 56 & 2830020 & 73 & 2973650 & 78 & 2997582 \\
\hline & 25 & 32 & 2086103 & 57 & 3183516 & 77 & 3545895 & 93 & 3687400 \\
\hline & 30 & 32 & 2086103 & 57 & 3398607 & 81 & 4148823 & 100 & 4317304 \\
\hline
\end{tabular}

results are presented in Table 1 , where $\eta_{s}^{\max }$ can vary from 5 to $30 \%$ of all slots available in a subdistrict $s$ and $\gamma_{s}^{\max }$ may vary from 5 to $20 \%$ of the total number clusters in a subdistrict $s$ (note that in the table we express such values as fraction of decimal numbers). Each entry of the table reports two values for the best solution: the first is the number of activated clusters, whereas the second is the total profit granted by the solution (reported in EUR).

In the solutions obtained for the model BLP-PS for this instance, we can note that there is a tendency to push the number of rented clusters to the dominating upper bound $\eta_{s}^{\max }$ or $\gamma_{s}^{\max }$ : the objective function pursues the activation of as many large clusters as possible, presenting the highest profit value, and this is jointly upper bounded by $\gamma_{s}^{\max }$, the bound on the number of clusters, and by $\eta_{s}^{\max }$, the bound on the total number of parking slots $\eta_{s}^{\max }$. So, for example, when considering an upper bound of $5 \%$ on the number of activated slots, the associated profit remains the same for an upper bound on the number of activated clusters higher or equal than $10 \%$, indicating that in this case is the bound on the number of clusters to dominate the other. In contrast, for example, an upper bound on the number of activated clusters equal to $5 \%$ dominates that on the number of slots for a percentage higher or equal than $10 \%$.

Looking at Table 1, we can notice that the number of activated clusters can sensibly increase as the upper bounds on the number of rentable clusters and slots increase. In particular, we can pass from a minimum of 28 activated clusters granting a profit of about $800 \mathrm{k}$ EUR, in the case of the lowest 
combination of bounds, to a maximum of 102 clusters associated with a profit that is more than 5 times higher, namely about 4300k EUR, in the case of the highest combination of bounds. For intermediate bound combinations, the number of activated clusters and associated profit vary quite regularly, thus giving the possibility to the LG to adjust the bounds in order to pursue the target of cluster activation that better suits its objective for renting to carsharing services.

Finally, we conducted computational tests on a set of 20 data instances presenting an increasing number of districts, subdistricts and clusters, with the number of clusters ranging from 478 to 3428 and the number of total available slots ranging from 1578 to 17140 . We compared the performance of our GA-based matheuristic to that of the solver CPLEX, used to solve the model BLP-PS with a time limit of 3600 seconds (the time budget assigned to CPLEX is thus equal to the total time budget assigned to the matheuristic). As upper bounds $\gamma_{s}^{\max }$ and $\eta_{s}^{\max }$, we adopted $20 \%$ of the total number of available slots and $15 \%$ of the total number of available clusters.

The results of our tests are reported in Table 2, where: a) ID is the instance identification; 2) $\Sigma s$ is the total number of available clusters in the instance; 3) $\Sigma n$ is the total number of available parking slots in the instance; 4) $\Sigma n^{\mathrm{MH}}$ is the total number of activated parking slots in the best solution found by our MatHeuristic (denoted by the acronym $\mathrm{MH}$ ) within the time limit; 5) $\pi^{\mathrm{MH}}$ is the total profit (expressed in EUR) associated with the best solution found by $\mathrm{MH}$ within the time limit; 6) $t^{\mathrm{MH}}$ is the time (expressed in seconds) required by $\mathrm{MH}$ to find the best solution within the time limit; 7) $\Sigma n^{\text {CPLEX }}$ is the total number of activated parking slots in the best solution found by CPLEX within the time limit; 8) $\pi^{\text {CPLEX }}$ is the total profit (expressed in EUR) associated with the best solution found by CPLEX within the time limit; 9) $t^{\text {CPLEX }}$ is the time (expressed in seconds) required by CPLEX to find an optimal solution (when an optimal solution is not found within the time limit, we insert the time limit of 3600 seconds as value);10) $\Delta \pi \%$ is the percentage of profit increase that MH grants with respect to CPLEX. In Table 2, the values in the columns $\pi^{\mathrm{MH}}$ and $\pi^{\mathrm{CPLEX}}$ include an asterisk $\left(^{*}\right)$ when the value is optimal for the instance.

Comparing the performance of our matheuristic MH and CPLEX, we can subdivide the instances in three subsets on the basis of the results obtained:

- in the case of the smaller instances from I1 to I6, both MH and CPLEX find the optimal solution. However, CPLEX is faster and MH takes on average $18 \%$ more time to identify the best solution;

- also in the case of the instances from I7 to I14, both MH and CPLEX 
find the optimal solution, but $\mathrm{MH}$ is faster and reduces the computational time of about $12 \%$ on average;

- in the case of the larger instances from I15 to I20, CPLEX does not identify an optimal solution within the time limit. Moreover, for all these instances, the value of the best solution found by CPLEX is lower than that of the best solution found by MH. Specifically, the best solutions found by $\mathrm{MH}$ offer an increased profit that is about $10 \%$ higher on average. Finding such better solutions requires a consistent fraction of the available time budget.

For all the instances, it is interesting to note that, in general, it is the bound on the number of available slots to lead the cluster activation, pushing towards the selection of a subset of clusters that respect the bound $\eta_{s}^{\max }$ and that are as large as possible, so to grant the highest profit for single slot (we recall indeed that larger clusters are preferred by carsharing companies, since they grant more visibility to the service, and are thus associated with a higher renting price per single slot composing the cluster). It is also interesting to note that, for all instances, while the number of activated slots remains around $20 \%$ of the total number of available slots, the profit sensibly increases passing from the smallest to the largest instance: this is due to the fact that in larger instances there is the possibility of activating a higher number of clusters of larger size, which, as discussed, are more attractive and their rent grants a remarkably higher profit per single slot than clusters of smaller size.

\section{Discussion}

Concerning the real-world adoption and implementation of the original optimization approach we proposed, we believe that a crucial first challenge is represented by convincing local governments of the advantages that a rigorous mathematical optimization approach may offer with respect to trialand-error approaches that we have often observed among professionals in the field of urban mobility and transportation systems. Several recent works have clearly shown the advantages of adopting mathematical optimization to solve real-world optimization problems on behalf of companies, also in the transportation field (see e.g., Borndörfer et al. 2018; Fujisawa et al. 2016). Referring in particular to the case of carsharing, reserved parking slots are not a novelty, but, to the best of our knowledge, a mathematical optimization model has never been proposed for the selection of the slots among a set of candidate sites. In most of the cases, the reserved slots are 
Table 2: Results for instances of increasing size

\begin{tabular}{ccc|ccc|ccc|c}
\hline ID & $\boldsymbol{\Sigma} \boldsymbol{c}$ & $\boldsymbol{\Sigma} \boldsymbol{n}$ & $\boldsymbol{\Sigma} \boldsymbol{n}^{\mathrm{MH}}$ & $\boldsymbol{\pi}^{\mathrm{MH}}$ & $\boldsymbol{t}^{\mathrm{MH}}$ & $\boldsymbol{\Sigma}^{\text {CPLEX }}$ & $\boldsymbol{\pi}^{\text {CPLEX }}$ & $\boldsymbol{t}^{\text {CPLEX }}$ & $\boldsymbol{\Delta} \boldsymbol{\pi} \boldsymbol{\%}$ \\
\hline I1 & 478 & 1578 & 307 & $1350349^{*}$ & 343 & 307 & $1350349^{*}$ & 259 & 0.00 \\
I2 & 562 & 1967 & 392 & $2085164^{*}$ & 468 & 392 & $2085164^{*}$ & 406 & 0.00 \\
I3 & 601 & 2164 & 422 & $2701938^{*}$ & 650 & 422 & $2701938^{*}$ & 513 & 0.00 \\
I4 & 639 & 2109 & 423 & $2164170^{*}$ & 602 & 423 & $2164170^{*}$ & 538 & 0.00 \\
I5 & 776 & 2484 & 492 & $3094116^{*}$ & 764 & 492 & $3094116^{*}$ & 676 & 0.00 \\
I6 & 802 & 2807 & 551 & $3910623^{*}$ & 681 & 551 & $3910623^{*}$ & 629 & 0.00 \\
I7 & 932 & 3542 & 684 & $4705261^{*}$ & 613 & 684 & $4705261^{*}$ & 680 & 0.00 \\
I8 & 949 & 3702 & 737 & $5326327^{*}$ & 756 & 737 & $5326327^{*}$ & 846 & 0.00 \\
I9 & 1047 & 4188 & 837 & $5391653^{*}$ & 852 & 837 & $5391653^{*}$ & 892 & 0.00 \\
I10 & 1122 & 4713 & 942 & $5654585^{*}$ & 877 & 942 & $5654585^{*}$ & 1007 & 0.00 \\
I11 & 1367 & 5742 & 1117 & $6527808^{*}$ & 1492 & 1117 & $6527808^{*}$ & 1873 & 0.00 \\
I12 & 1492 & 6267 & 1235 & $7911311^{*}$ & 2285 & 1235 & $7911311^{*}$ & 2642 & 0.00 \\
I13 & 1643 & 7394 & 1465 & $10138284^{*}$ & 1964 & 1465 & $10138284^{*}$ & 2365 & 0.00 \\
I14 & 1828 & 8226 & 1642 & $10691682^{*}$ & 2554 & 1642 & $10691682^{*}$ & 2911 & 0.00 \\
I15 & 2156 & 9702 & 1849 & 11444247 & 3126 & 1720 & 10647578 & 3600 & 6.96 \\
I16 & 2382 & 10958 & 2072 & 13704218 & 3295 & 1896 & 12545038 & 3600 & 8.45 \\
I17 & 2505 & 11774 & 2326 & 14696307 & 3332 & 2140 & 13524295 & 3600 & 7.97 \\
I18 & 2891 & 13588 & 2638 & 16095856 & 3483 & 2387 & 14571878 & 3600 & 9.46 \\
I19 & 3125 & 14375 & 2681 & 16892588 & 3490 & 2386 & 15034753 & 3600 & 10.99 \\
I20 & 3428 & 17140 & 3233 & 21079811 & 3204 & 2861 & 18657918 & 3600 & 11.48 \\
\hline
\end{tabular}


simply placed close to major landmarks, like airports and railway stations, without an analytical approach supporting their selection and without in particular pursuing the fair distribution principle that we have formalized through the feasibility constraints (5)-(7).

Once the mathematical optimization approach is accepted as effective and efficient, the mobility officers of the local governments should then closely work together with the optimization experts: the aim would be to properly define a data input of the model that is meaningful and valid for the districts subject to carsharing parking planning. In this phase, the officers could also suggest additional (soft) constraints to be taken into account for defining a model and generating a data input that allow to better represent specific features of the considered districts. Within this phase, the risk of miscommunication between officers and experts is concrete, especially when the officers do not have an education or background that allow them to understand and express system modelling needs. In our recent experience, it seems that the situation is getting better year after year, thanks to the tendency to hire data science experts in various departments of local governments, allowing the optimization consultants to interface with officers who are more familiar with mathematical modelling and algorithmic approaches.

After having defined the input data, solved the model and obtained a carsharing parking slot plan, the local governments could still face conflicts during the implementation of the plan. In particular, referring to the Italian case that we better know also thanks to our direct consulting experience, the parking slots reserved to carsharing would further increase the number of slots that are subtracted to toll free parking and to the parking of district residents and this could lead to legal appeals by residents. In major Italian cities, substantial modifications to parking plans have regularly generated appeals, even reaching the Supreme Court of Cassation, which has ruled against them. To avoid this, we think that it is fundamental to orchestrate relevant changes to parking plans with local residents, involving them in the decision process. From the point of view of our optimization model, this would imply to involve the residents in defining the location and size of the candidate clusters and the values of the bounds that appear in constraints (5)-(7), which limit the number of slots that can be reserved to carsharing in districts and subdistricts.

\section{Conclusions and Future Work}

In this paper, we have characterized the optimization problem of a local government that wants to analytically choose the best subset of parking 
slots to rent to carsharing companies, with the aim of reducing cruising for finding parking and improve urban mobility. We proved that the problem is NP-Hard and we proposed a new Binary Linear Programming problem to model it. Given the NP-Hard nature of the problem, which can make it hard to solve even for state-of-the-art solvers, we proposed a new genetic-based matheuristic for its solution. The matheuristic combines a genetic algorithm with solution construction and improvement phases that are based on the execution of exact large neighborhood searches. We tested the performance of our new optimization approach on realistic instances referring to the city of Rome, showing that it can offer a better computational performance of a state-of-the-art solver and that it can return a fair distribution of clusters over the territory. As future work, we intend to define a multiperiod version of the model, to consider parking slot renting over long-term periods and include variations of demand for carsharing over time, also extending the computational tests to other cities. In this context, we would expect to face demand uncertainty, not knowing in advance the precise demand for carsharing services in the future, and thus it would be necessary to adopt optimization under data uncertainty methods, such as (Multiband) Robust Optimization (Büsing and D'Andreagiovanni, 2012). Furthermore, another topic that we consider worth of being investigated is how the clusters selected for renting should be assigned to carsharing companies. We expect that this should be done by means of public tenders, also taking into account fair competition criteria that favour the entry of new carsharing companies, similarly to the rules adopted by cities like Sydney - see (City of Sydney, 2016).

\section{References}

Becker, H., Ciari, F., Axhausen, K.W., 2018. Measuring the car ownership impact of free-floating car-sharing - a case study in Basel, Switzerland. Transport. Res. D 65, 51-62.

Bencardino, M., Greco, I., 2014. Smart Communities. Social Innovation at the Service of the Smart Cities. TeMA. J. Land Use Mob. Environ., INPUT 2014, 39-51.

Benevolo, C., Dameri, R.P., D'Auria, B., 2016. Smart Mobility in Smart City - Action Taxonomy, ICT Intensity and Public Benefits. In: T. Torre et al. (Eds.), Empowering Organizations, Lecture Notes in Information Systems and Organisation 11, Springer. 
Bertsimas, D., Tsitsiklis, J.N., 1997. Introduction to Linear Optimization. Athena Scientific, Belmont.

Biondi, E., Boldrini, C., Bruno, R., 2016. Optimal deployment of stations for a car sharing system with stochastic demands: A queueing theoretical perspective. IEEE 19th International Conference on Intelligent Transportation Systems (ITSC 2016), 1089-1095.

R. Borndörfer, T. Klug, L. Lamorgese, C. Mannino, M. Reuther, T. Schlechte: Handbook of Optimization in the Railway Industry. Springer, Cham (2018)

Boyaci, B., Zografos, K.G., Geroliminis, N., 2017. An integrated optimization-simulation framework for vehicle and personnel relocations of electric carsharing systems with reservations, Transport. Res. B 95, 214-237.

Blum, C., Puchinger, J., Raidl, G.R., Roli., A., 2011. Hybrid metaheuristics in combinatorial optimization: a survey. Applied Soft Computing 11(6), $4135-4151$.

Bruglieri, M., Pezzella, F., Pisacane, O., 2018. A two-phase optimization method for a multiobjective vehicle relocation problem in electric carsharing systems. J. Comb. Optim. 36, 162-193.

Büsing, C., D'Andreagiovanni, F.: New results about Multi-band Uncertainty in Robust Optimization. In: Klasing, R. (ed.) Experimental Algorithms - SEA 2012, Lecture Notes in Computer Science, vol. 7276, pp. 63-74. Springer, Heidelberg (2012)

Carrese, S., D'Andreagiovanni, F., Giacchetti, T., Nardin, A., Zamberlan, L. 2020. An optimization model for renting public parking slots to carsharing services. Transportation Research Procedia 45, 499-506.

Carrese, S., Giacchetti, T., Nigro, M., Patella, S.M., 2017. An innovative car sharing electric vehicle system: an italian experience. WIT Transactions on The Built Environment 176, 245-252.

Carrese, S., Gori, S., Picano, T., 1996. Relationship between parking location and traffic flows in urban areas. Advanced Methods in Transportation Analysis, 183-214, Springer. 
Chen, X., Cheng, J., Ye, J., Jin, Y., Li, X., Zhang, F., 2018. Locating Station of One-Way Carsharing Based on Spatial Demand Characteristics. J. Adv. Transport. 2018, ID 5493632.

Cheng, Y., Chen, Ding, X., Zeng, L., 2019. Optimizing Location of CarSharing Stations Based on Potential Travel Demand and Present Operation Characteristics: The Case of Chengdu. J. Adv. Transport. 2019, ID 7546303.

City of Ashfield, 2010. Sustainability Action Table. http://www.ashfield.nsw.gov.au/files//your_council/policies_plans_and_reports/ policies/Ashfield_Sustainability_Action_Table_2010.pdf

City of Calgary. 2011. Bill number TP017 (A Parking Policy Framework for Calgary). http://www.calgary.ca/CA/city-clerks/Documents/Councilpolicy-library/TP017-ParkingPolicyFramework.pdf

City of Milan, 2016. Deliberazione Giunta Comunale n.55 (in Italian). https://www.comune.milano.it/dseserver/webcity/garecontratti.nsf/ 51607b595b240841c1256c4500569c90/fb7227ebfe458120c1257fa900593003/\$FILE /ATTS0RDL.pdf/Determina\%20-\%2020160427_55\%20\%20Bando\%20CarSharing\%202016.pdf

City of Rome, 2016. Deliberazione Giunta Comunale n.136 (in Italian). http://www.comune.roma.it/servizi/DeliberazioniAttiWeb/

City of Sidney, 2016. Council Meeting Notice No. 10/1579 http://www.cityofsydney.nsw.gov.au/_data/assets/pdf_file/0007/277738/161121 _COUNCIL_MINUTES.pdf.

City of Turin, 2015. Determinazione Dirigenziale n.46 (in Italian) http://www.comune.torino.it/giunta_comune/intracom/htdocs/2015/2015_00248.pdf.

City of Vancouver, 2016.Bill number 11591 (Street and traffic regulation in the city of Vancouver) http://bylaws.vancouver.ca/2849c.pdf

D'Andreagiovanni, F., Krolikowski, J., Pulaj, J., 2015. J. A fast hybrid primal heuristic for Multiband Robust Capacitated Network Design with multiple time periods. App. Soft Comp. 26, 497-507.

D'Andreagiovanni, F., Nardin, A., 2015. J. Towards the fast and robust optimal design of wireless body area networks. App. Soft Comp. 37, 971982. 
Danna, E., Rothberg, E., Le Pape, C. 2005. Exploring relaxation induced neighborhoods to improve MIP solutions. Math. Program. 102, 71-90.

Dowling, R., Kent, J., 2015. Practice and public-private partnerships in sustainable transport governance: The case of car sharing in Sydney, Australia. Transport Policy 40.3, 58-64.

European Union Innovation and Networks Executive Agency (EU-INEA), 2017. Smart Cities \& Communities . https://ec.europa.eu/inea/en/horizon-2020/smart-cities-communities

K. Fujisawa, Y. Shinano, H. Waki: Optimization in the Real World. Springer, Cham (2016)

Global e-Sustainability Initiative (GeSI) and Accenture Strategy, 2015. \#SMARTer2030 ICT Solutions for 21 st Century Challenges. http://smarter2030.gesi.org/downloads/Chapter_Mobility.pdf

Hessel, V., 2015. Smart Mobility - A tool to achieve sustainable cities. Siemens Mobility Division. http://www.vt.bgu.tum.de/fileadmin/w00bnf/www/VKA/2014_15/150212_Smart_Mobility_v5_TUN

Goldberg, D.E., 1988. Genetic Algorithms in Search, Optimization \& Machine Learning. Addison-Wesley, Reading.

IBM ILOG CPLEX, http://www-01.ibm.com/software/integration/optimization/cplexoptimizer.

Illgen, S., Höck, M., 2019. Literature review of the vehicle relocation problem in one-way car sharing networks, Transport. Res. B, 120, 193-204.

Karakatic, S., Podgorelec, V., 2015. A survey of genetic algorithms for solving multi depot vehicle routing problem. Applied Soft Computing 27, 519 -532 .

Kenny, P., 2013. Smart Mobility for Smart Cities European Policy and Practice. IBTTA Global Summit, https://ibtta.org/sites/default/files/documents/2014/14Prague/Kenny_Padraig.pdf

Li, W., Li, Y., Fan, J., Deng, H., 2017. Siting of carsharing stations based on spatial multi-criteria evaluation: A case study of Shanghai EVCARD. Sustainability $9(1), 152$. 
Lombard, P.L., Molocchi, A., Buscema, I., Molinario, G., 2005. I costi ambientali e sociali della mobilitá in Italia - Quinto Rapporto (in Italian). Ferrovie dello Stato, http://www.emrg.it/Lezioni_Energia_Rinnovabile/V\%20Rapporto\%20italiano.pdf

Lyons, G., 2016. Getting smart about urban mobility - Aligning the paradigms of smart and sustabinable. Transport. Res. A., 115, 4-14.

Maniezzo, V, 1999. Exact and Approximate Nondeterministic Tree-Search Procedures for the Quadratic Assignment Problem. INFORMS J. Comp. 11 (4) 358-369.

Maniezzo, V., Stüzle, T., Voß, S., 2009. Matheuristics: Hybridizing Metaheuristics and Mathematical Programming. Springer, Heidelberg.

Martin, E.W., Shaheen, S.A., 2011. Greenhouse gas emission impacts of carsharing in North America. IEEE Trans. Intell. Transp. Syst. 12.4, 10741086 .

Meisel, S., Merfeld, T., 2018. Economic incentives for the adoption of electric vehicles: A classification and review of e-vehicle services. Transport. Res. D 65, 264-287.

Millard-Ball, A., Schipper, L., 2011. Are we reaching peak travel? Trends in passenger transport in eight industrialized countries. Transport Reviews $31,357-378$.

Motwani, R., Raghavan, P., 1995. Randomized algorithms. Cambridge University Press, New York.

New York City Council, 2015. A Local Law to amend the administrative code of the city of New York, in relation to establishing a carsharing parking pilot program. http://legistar.council.nyc.gov/LegislationDetail.aspx?ID=2404408\&GUID=090E4AA83B4E-4B66-95B5-DB3B5B69C1D1

Newman, P., Kenworthy, J., 2011. Peak car use: Understanding the demise of automobile dependence. World Transp. Policy \& Prac. 17, 31-39.

Pinna, F., Masala, F., Garau, C., 2017. Urban Policies and Mobility Trends in Italian Smart Cities. Sustainability 9(4), 1-21.

Rivasplata, C., Guo, Z., Lee, R., Keyon, D., 2013. Residential On-Site Carsharing and Off-Street Parking in the San Francisco Bay Area, California. Transp. Res. Record 2359, 68-75. 
Shaheen, S., Cohen, A., Martin, E., 2010. Carsharing Parking Policy - Review of North American Practices and San Francisco, California, Bay Area Case Study. Transp. Res. Record 2187, 146-156.

Srinivas, M., Patnaik, L.M, 1994. Genetic algorithms: a survey. Computer $27(6), 17-26$.

Wang, H., Zhao, D., Meng, Q., Ong, G.P., Lee, D., 2019. A four-step method for electric-vehicle charging facility deployment in a dense city: An empirical study in Singapore, Transport. Res. A 119, 224-237.

Weikl, S., Bogenberger, K., 2013. Relocation Strategies and Algorithms for Free-Floating Car Sharing Systems. IEEE Intell. Transp. Syst. Mag. 5.4, 100-111.

Wu, C., Le Vine, S., Sivakumar, A., Polak, J., 2019. Traveller preferences for free-floating carsharing vehicle allocation mechanisms. Transport. Res. C 102, 1-19.

$\mathrm{Xu}$, Z., 2011. The knapsack problem with a minimum filling constraint. Naval Research Logistics 60(1), 56-63.

Zhao, M., Li, X., Yin, J., Cui, J., Yang, L., An, S., 2018. An integrated framework for electric vehicle rebalancing and staff relocation in one-way carsharing systems: Model formulation and Lagrangian relaxation-based solution approach, Transport. Res. B 117, 542-572. 